\title{
The Effectiveness of Hand Massage on Pain in Critically III Patients After Cardiac Surgery: A Randomized Controlled Trial Protocol
}

Madalina Boitor ${ }^{1}, \mathrm{RN}$; Géraldine Martorella ${ }^{2}, \mathrm{RN}, \mathrm{PhD}$; Andréa Maria Laizner ${ }^{1}, \mathrm{RN}, \mathrm{PhD}$; Christine Maheu ${ }^{1}, \mathrm{RN}$, $\mathrm{PhD}$; Céline Gélinas ${ }^{1}, \mathrm{RN}, \mathrm{PhD}$

\footnotetext{
${ }_{1}^{1}$ Ingram School of Nursing, Faculty of Medicine, McGill University, Montreal, QC, Canada

${ }^{2}$ College of Nursing, Florida State University, Florida, FL, United States
}

Corresponding Author:

Madalina Boitor, RN

Ingram School of Nursing

Faculty of Medicine

McGill University

3506 University

Montreal, QC

Canada

Phone: 15143984144

Fax: 15143988455

Email: madalina.boitor@mail.mcgill.ca

\section{Abstract}

Background: Postoperative pain is common in the intensive care unit despite the administration of analgesia. Some trials suggest that massage can be effective at reducing postoperative pain in acute care units; however, its effects on pain relief in the intensive care unit and when pain severity is highest remain unknown.

Objective: The objective is to evaluate the effectiveness of hand massage on the pain intensity (primary outcome), unpleasantness and interference, muscle tension, anxiety, and vital signs of critically ill patients after cardiac surgery.

Methods: A 3-arm randomized controlled trial will be conducted. A total of 79 patients who are 18 years or older, able to speak French or English and self-report symptoms, have undergone elective cardiac surgery, and do not have a high risk of postoperative complications and contraindications to hand massage will be recruited. They will be randomly allocated (1:1:1) to standard care plus either 3 20-minute hand massages (experimental), 3 20-minute hand holdings (active control), or 320 -minute rest periods (passive control). Pain intensity, unpleasantness, anxiety, muscle tension, and vital signs will be evaluated before, immediately after, and 30 minutes later for each intervention administered within 24 hours postoperatively. Peer-reviewed competitive funding was received from the Quebec Nursing Intervention Research Network and McGill University in December 2015, and research ethics approval was obtained February 2016.

Results: Recruitment started in April 2016, and data collection is expected to be complete by January 2017 . To date, 24 patients were randomized and had data collection done.

Conclusions: This study will be one of the first randomized controlled trials to examine the effect of hand massage on the pain levels of critically ill patients after cardiac surgery and to provide empirical evidence for the use of massage among this population.

ClinicalTrial: ClinicalTrials.gov NCT02679534; https://clinicaltrials.gov/ct2/show/NCT02679534 (Archived by WebCite at http://www.webcitation.org/618Ly5eHS)

(JMIR Res Protoc 2016;5(4):e203) doi: 10.2196/resprot.6277

\section{KEYWORDS}

massage; pain; critical care; randomized controlled trial; anxiety; muscle tension; vital signs; clinical protocol; complementary therapies; thoracic surgery 


\section{Introduction}

\section{Overview}

Undergoing cardiac surgery constitutes a major event for patients that is accompanied by physical and psychological symptoms such as postoperative pain [1-5] and anxiety [6-8]. Recent studies reveal that massage could complement pharmacological treatments and have positive effects in reducing these symptoms in acute care units [9-11], yet empirical evidence is lacking to support the same effects early in the postoperative phase when patients are in the intensive care unit (ICU) and pain is at its highest.

In the ICU, postoperative pain can be compounded by routine ICU procedures such as turning, coughing, breathing, and chest tube removal, activities which are perceived to be the most painful in the immediate postoperative period $[3,5]$. Given the higher severity and complexity of pain in the ICU, findings from massage studies conducted on acute care wards cannot be extrapolated to the unique context of the ICU and the early recovery phase after cardiac surgery. Further evidence is needed to unravel the potential role of massage in relieving the pain of cardiac surgery in ICU patients and guide international clinical practice guidelines with regard to the use of this complementary nonpharmacological intervention in this patient population.

\section{Background}

Cardiac surgeries, such as coronary artery bypass grafting and valve replacement, rank among the most frequently performed surgical interventions worldwide [12] and necessitate the routine admission of patients to the ICU. Cardiac surgeries are commonly indicated to reduce anginal pain, but the surgical procedure itself can lead to the development of postoperative pain. Mounting evidence shows that cardiac surgery ICU patients experience moderate-to-severe pain reaching proportions as high as 74\% despite the use of analgesics [2-5], with the highest pain intensity commonly experienced in the first 24 hours postsurgery [13].

Unrelieved postoperative pain can interfere with patients' ability to cough and mobilize effectively, which predisposes them to postoperative complications such as atelectasis, pneumonia, and deep vein thrombosis $[2,5,14]$, thereby delaying recovery and ICU discharge. Moreover, the intensity of acute postoperative pain immediately after surgery is a significant predictor of the presence and severity of persistent postoperative pain up to 2 years postsurgery [15-17], a serious and often unrecognized complication after cardiac surgery that may interfere with daily activities and quality of life [18].
Among the pharmacological approaches to pain control, opioids constitute the mainstay of treatment in the ICU $[19,20]$, yet pain has been shown to persist even during unrestricted use of these analgesic agents [13,21]. The use of complementary nonpharmacologic interventions such as massage has been suggested in the clinical practice guidelines of the Society of Critical Care Medicine given their opioid-sparing and analgesia-enhancing potential [20]. Massage has been defined as the manual manipulation of muscles and soft tissues of the body through the application of various systematic and rhythmic hand movements [22,23].

A recent systematic review exploring the effectiveness of massage on postoperative outcomes among patients undergoing cardiac surgery [24] indicated that out of the 7 eligible studies ( $\mathrm{N}=40-252 /$ study), 6 reported that massage therapy ranging from 20 to 30 minutes in duration improved postoperative outcomes such as pain, anxiety, and muscle tension [9,10,25-28], while only one study reported no positive results [29]. The latter study evaluated the effectiveness of a 30-minute massage therapy involving each limb for 5 minutes followed by a 10-minute back massage while patients were lying on the left side. The positioning required for the back massage might have obscured or minimized the potential benefit of massage in relieving pain, anxiety, and muscle tension given that side-lying may exert traction on the sternotomy site, causing pain [3] and increasing muscle tension and possibly anxiety as turning on one side could be perceived as a painful procedure. Of note, only one of these studies was conducted in the ICU [25], and it lacked random assignment.

One pilot randomized controlled trial (RCT) conducted with 40 ICU patients post-cardiac surgery [30] showed promising pain relief and muscle relaxant effects of up to 3 15-minute hand massages, whereas the administration of a single massage therapy did not yield any significant decrease in pain intensity or muscle tension, suggesting that repeated administration of hand massage is necessary in this patient population.

Overall, there is a paucity of high-level evidence on which to base massage therapy decisions in the management of pain in post-cardiac surgery ICU patients. Extrapolations of evidence from other patient populations and clinical settings are flawed by the differing health status and symptom severity of this specific subgroup of ICU patients. Future rigorous RCTs conducted in the context of the ICU and with cardiac surgery adults in their immediate postoperative period are essential to make recommendations for the use of massage in clinical practice including the minimal effective dose, body area massaged, and techniques employed. 
Figure 1. Adapted diagram of the neuromatrix theory of pain.

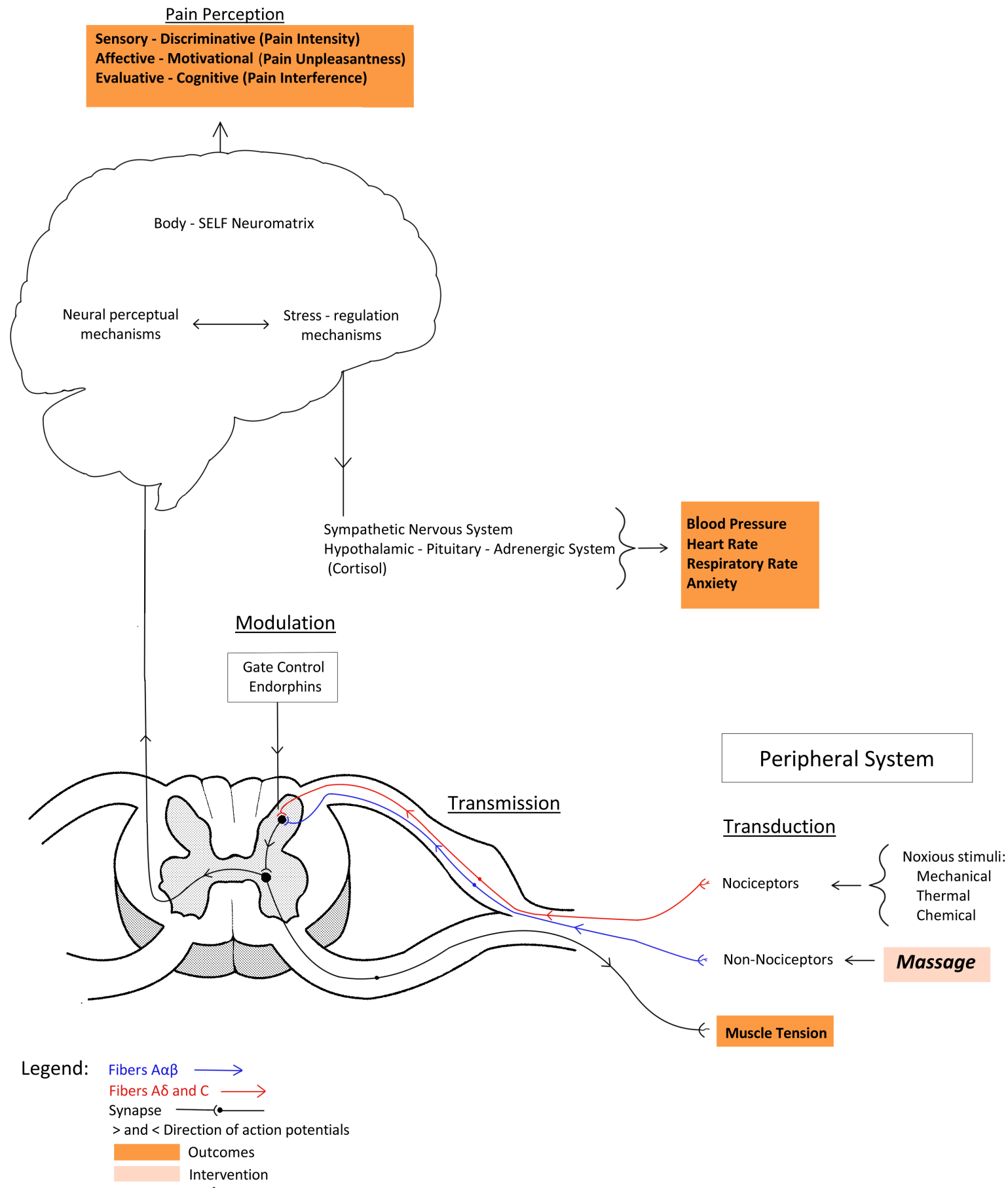


Textbox 1. Eligibility criteria.

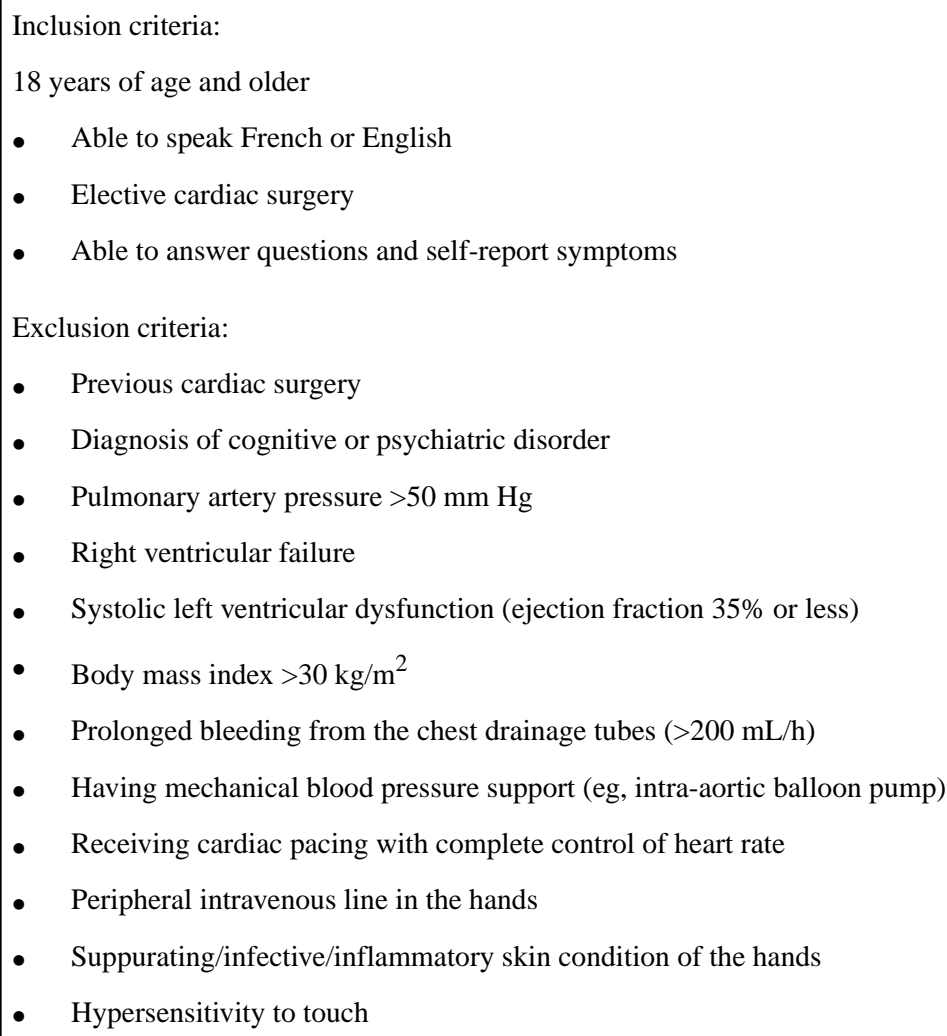

\section{Theoretical Framework}

The protocol is based on the neuromatrix theory of pain [31] where the brain's neural network, the body-self neuromatrix, integrates multiple inputs such as sensory (eg, cutaneous) and opioid systems (ie, endogenous opioids) to influence the sensory (intensity, localization, and quality), affective (unpleasantness), and cognitive dimensions (interference with daily functioning) of pain (Figure 1). The sensory stimulation of nonnociceptive fibers in the skin and muscles involved in massage could block the transmission of nociceptive impulses in the dorsal horns and may increase the release of beta-endorphins in the bloodstream [32,33], which block the release of neurotransmitters from the nociceptive fibers (especially substance P) [34], thereby blocking the transmission of nociceptive impulses from reaching the body-self neuromatrix where pain perception occurs. Similarly, by blocking the transmission of nociceptive signals, the stimulation of the stress-regulation system responsible for the activation of the noradrenergic and sympathetic nervous system could be inhibited. The resultant lack of up-regulation of adrenaline and noradrenaline could explain the potential effects of massage in decreasing blood pressure, heart and respiratory rates, and the subjective sensations of anxiety [35-37].

\footnotetext{
Aim

The primary aim of this research study is to compare the effect of 320 -minute hand massage administrations by a trained nurse within 24 hours after cardiac surgery versus hand-holding (ie, simple touch) and standard care on the postoperative pain intensity of adult ICU patients.
}

\section{Methods}

\section{Trial Design}

This research study is designed as a randomized, controlled, patient-blinded, single-center superiority trial with 3 parallel groups and a 1:1:1 allocation ratio. A modified Consolidated Standards of Reporting Trials flow diagram for individual RCTs of nonpharmacologic treatments will be used to document recruitment and retention of participants (see Multimedia Appendix 1) [43].

\section{Participants}

This RCT targets adults admitted to the ICU after undergoing cardiac surgery in a university-affiliated hospital in Canada. A single setting was selected for this study because of standardized pain management practices and surgical techniques and single patient rooms. Patients will be screened for inclusion using the eligibility criteria seen in Textbox 1. Patients at higher risk of postoperative complications and those with contradictions to having their hands massaged will be excluded.

\section{Sample Size and Sampling Procedure}

A power analysis was conducted using the $\mathrm{G}^{*}$ Power 3 program [38] to estimate the sample size required to capture the potential effects of massage to decrease the primary outcome (pain intensity) and to strengthen the statistical conclusion validity. The mean treatment difference was observed to be greater than 1.5 in several RCTs $[11,26,39,40]$ and approximates the clinically significant difference of 2 points on the 0 to 10 Numeric Rating Scale (NRS) [41]. This trial is powered to be able to detect a difference in the pain intensity score of 1.5 
between the hand massage and standard care group. The standard deviation of pain intensity scores is approximately a 2.0 value, which was selected for the sample size calculation. To detect a mean difference in pain intensity scores of 1.5 points (SD 2.0) immediately after the third massage with a 2-sided significance level of .05 and power of .80 with equal allocation to 3 arms and a repeated measures between factors context with 3 measurements would require a minimum of 72 patients.

Given the low attrition rates observed with this patient population $(0 \%[9,10,30]$ and $35 / 287(12.2 \%)$ [29]), a $10 \%$ drop-out rate is considered in the calculation of the total sample size. Therefore, the final size required for this RCT is 79, and it will be reached using convenience sampling.

\section{Randomization}

Before the study begins, permuted-block randomization will be generated for 85 patients by a statistician using SAS computer software (SAS Institute) and block sizes of 3 , an allocation ratio of $1: 1: 1$, and one strata to minimize the imbalance in the number allocated to each group. Then, the randomization schedule will be transcribed in sequentially numbered and opaque sealed envelopes by a research coordinator not involved in assignment allocation to ensure allocation concealment. The allocation list will be stored in a locked filing cabinet of the principal investigator and will not made accessible to the interventionist involved in enrollment and treatment allocation.

\section{Recruitment of Participants}

Patient recruitment will begin preoperatively when eligibility criteria such as age and language spoken will be verified (Table 1).

The remaining eligibility criteria (eg, blood loss, peripheral intravenous lines) will be evaluated post-cardiac surgery and ICU admission. After the collection of baseline data, patients meeting all the inclusion criteria will be randomly assigned to either hand massage, hand-holding, or standard care. As each participant enters the study, he or she receives the next envelope in the sequence, thereby concealing the interventionist's and trial participants' knowledge of the upcoming group assignment.

The interventionist will then administer the assigned intervention (hand massage, hand-holding) without informing participants of their group assignment until the end of data collection. Nurses and other ICU clinicians will also be masked to patients' group assignment. The similarity of the hand massage and hand-holding therapy characteristics serves to mask study participants and clinicians with regard to the specific intervention received as observed in a feasibility and acceptability study where patients receiving hand-holding referred to the intervention as massage [42]. Conversely, patients in the rest group are less likely to be masked to the group assigned.

A modified Consolidated Standards of Reporting Trials flow diagram for individual RCTs of nonpharmacologic treatments will be used to document recruitment and retention of participants [43].

\section{Choice of Comparators}

The majority of massage studies include standard care control groups to examine the absolute efficacy of massage in improving outcome variables. While this is important in attributing benefits to the massage therapy itself, studies that involve the administration of massage by a trained therapist, as recommended in Cochrane Systematic Reviews [44], should equally include a touch control group to verify if the additional manipulation included in massage is superior to touch only. Some studies suggest that touch, a free and easily administered intervention not requiring training, can have potential pain relief effects [45] and should, thus, be included as an active control group in future RCT designs to additionally examine the relative efficacy of massage. Furthermore, the touch control group can help mask patients with regard to the group assigned through its resemblance with actual massage, thereby controlling for placebo effects [44].

\section{Interventions}

\section{Training of Interventionist and Timing of Interventions}

Eligible patients will be randomized in equal proportions between hand massage, hand-holding, and standard care. One interventionist will deliver the hand massage and hand-holding interventions, which will be standardized across participants. The interventionist is a registered nurse with no previous experience in massage therapy who will be trained by a professional massage therapist through an accredited workshop of 6 hours including practical exercises and verification of competency, as was done in the pilot RCT [30].

The first intervention (hand massage or hand-holding) will be delivered in the evening of the day of surgery and the remaining two interventions the day after when patients are still in the ICU. Overall, three interventions will be administered within 24 hours postoperatively over the course of two days.

\section{Experimental Group}

Patients assigned to the experimental group will receive a 20-minute hand massage by the interventionist in addition to the standard ICU care. Before administering the massage, a favorable environment will be created that promotes calmness such as dampening the light, reducing the alarm intensity, closing the curtains and door, and posting a "do not disturb" notice, and a comfortable positioning of the patient will be ensured $[9,30,46]$. After performing hand hygiene and explaining the procedure to the patient, the interventionist will hold each hand for 5-10 seconds and apply 5-10 mL of unscented hypoallergenic cream to both hands and wrists. The cream will be supplied by the interventionist and reserved for use within the research context only. The interventionist will then perform massage using moderate pressure and stroking and kneading techniques during 10 minutes on the palm and back of each hand as inspired by the procedure by Kolcaba et al [47] and developed with the support of an experienced massage therapist (Textbox 2). 
Table 1. Study timeline.

\begin{tabular}{|c|c|c|c|c|c|}
\hline Procedures & Preop & $\begin{array}{l}\text { POD }^{\mathrm{a}} 0 \\
\text { evening }\end{array}$ & $\begin{array}{l}\text { POD } 1 \\
\text { early evening }\end{array}$ & $\begin{array}{l}\text { POD } 1 \\
\text { late evening }\end{array}$ & POD 2 \\
\hline \multicolumn{6}{|l|}{ Recruitment } \\
\hline First eligibility screen & $\mathrm{x}$ & & & & \\
\hline Informed consent & $\mathrm{x}$ & & & & \\
\hline Second eligibility screen & & $\mathrm{x}$ & & & \\
\hline Randomization & & $\mathrm{x}$ & & & \\
\hline \multicolumn{6}{|l|}{ Interventions } \\
\hline Hand massage & & $\mathrm{x}$ & $\mathrm{x}$ & $\mathrm{x}$ & \\
\hline Hand-holding & & $\mathrm{x}$ & $\mathrm{x}$ & $\mathrm{x}$ & \\
\hline Rest period & & $\mathrm{x}$ & $\mathrm{x}$ & $\mathrm{x}$ & \\
\hline Standard care & $\mathrm{x}$ & $\mathrm{x}$ & $\mathrm{x}$ & $\mathrm{x}$ & $\mathrm{x}$ \\
\hline \multicolumn{6}{|l|}{ Data collection } \\
\hline Demographics questionnaire & $\mathrm{x}$ & $\mathrm{x}$ & & & \\
\hline Pain intensity & & $\mathrm{x}$ & $\mathrm{x}$ & $\mathrm{x}$ & \\
\hline Pain unpleasantness & & $\mathrm{x}$ & $\mathrm{x}$ & $\mathrm{x}$ & \\
\hline Pain interference & & & & & $\mathrm{x}$ \\
\hline Pain location and quality & & $\mathrm{x}$ & $\mathrm{x}$ & $\mathrm{x}$ & \\
\hline Anxiety & & $\mathrm{x}$ & $\mathrm{x}$ & $\mathrm{x}$ & \\
\hline Muscle tension & & $\mathrm{x}$ & $\mathrm{x}$ & $\mathrm{x}$ & \\
\hline Vital signs & & $\mathrm{x}$ & $\mathrm{x}$ & $\mathrm{x}$ & \\
\hline
\end{tabular}

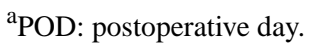

\section{Active Control Group}

The active control group will receive hand-holding by the same interventionist in addition to standard ICU care. The same hand hygiene and environmental adjustments will be made as for those receiving massage. Patients will have their hands held for 5-10 seconds and unscented hypoallergenic cream applied to both hands. Then, the interventionist will hold each of the patients' hand in her hand for ten minutes with occasional stroking for a total of 20 minutes.

\section{Passive Control Group}

The passive control group will receive the standard care administered in the ICU including a 20-minute rest period including the environmental adjustments of the experimental and active control groups. The interventionist will be outside of the patient room and have no contact with the patient throughout the 20 minutes. The standard care includes the pharmacological and nonpharmacological treatments (eg, repositioning) used to promote recovery and pain relief. In the study ICU, cardiac surgery patients are automatically prescribed a pain management protocol that includes the regular administration of morphine and breakthrough doses of analgesia as needed.

\section{Criteria for Modifying Interventions}

The allocated interventions will be discontinued upon the participant's withdrawal of consent or if skin irritation is suspected or patient comfort is disrupted due to the intervention itself, both of which will be reported as adverse events. Consenting participants will be retained in the trial whenever possible in spite of the discontinuation of the assigned intervention to allow remaining data collection and limit missing data.

\section{Concomitant Care}

Concomitant interventions may be received by patients while participating in this trial. Consenting patients will be permitted to receive any of the pharmacological treatments prescribed by their treating physician and any of the nonpharmacological interventions offered in the ICU (eg, back rub), and such data will be recorded. It is not prohibited that patients participate concomitantly in other research studies unless it involves any form of complementary therapy.

\section{Outcomes}

\section{Primary Outcome}

Pain intensity is the primary outcome and will be captured using the 0 to 10 NRS score. The analysis metric will be the change in pain intensity from baseline (preintervention) to immediately after each intervention and 30 minutes later. 
Textbox 2. Hand massage routine protocol.

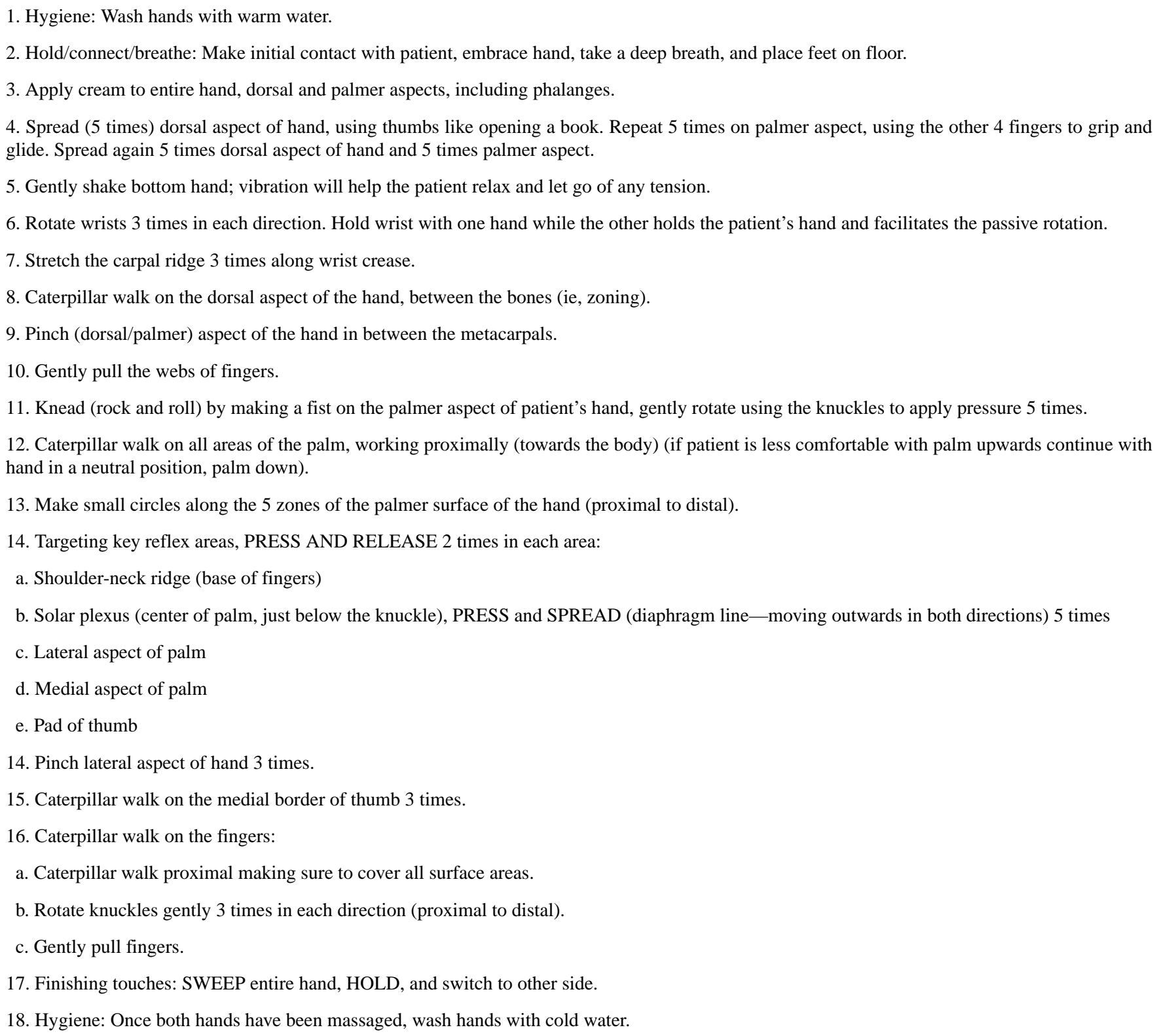

\section{Secondary Outcomes}

Pain unpleasantness, pain interference, muscle tension, anxiety, and vital signs will also be assessed in relation to each intervention, and means and standard deviations will be reported and used for data analysis.

\section{Instrumentation}

\section{Pain Intensity}

The NRS will be used to assess pain intensity. The NRS is an 11-point unidimensional self-report scale recommended for the assessment of pain intensity where 0 is no pain and 10 the worst possible pain. Details about the NRS and other assessment tools are summarized in Table 2. 
Table 2. Description and psychometrics of the instruments used for outcome data collection.

\begin{tabular}{lll}
\hline Outcome & Instrument & Scoring \\
& NRS $^{\mathrm{a}}(0-10)$ & 0 : no pain, \\
Pain & 10 : worst possible pain \\
intensity & \\
& & \\
& & \\
& & \\
Pain & NRS $(0-10)$ & $0:$ not at all unpleasant, \\
unpleasant- & & 10 most unpleasant feeling \\
ness & & possible
\end{tabular}

Psychometrics

Reliability

High test-retest reliability observed in cancer patients when measuring pain exacerbations (kappa=.86) and background pain (kappa $=.80)$ [48]. possible

Pain interference Adapted $\mathrm{BPI}^{\mathrm{b}}$ : pain intensity index (4 NRS 010 subscales), pain interference index (7 NRS 0 -10 subscales)

Muscle tension
$\mathrm{CPOT}^{\mathrm{C}}$ muscle tension subscale $(0-2)$
Pain intensity: 0: no pain,

10: pain as bad as you can imagine

Pain interference: 0: no interference, 10: interferes completely

0: no resistance,

1: resistance,

2: strong resistance
Internal consistency was also supported for this patient population with Cronbach alpha coefficients .84-.89 for the severity scale and .91-.94 for the interference scale.

Moderate to high interrater reliability of CPOT scores between trained raters with intraclass correlation of $0.30-0.86$ [52] and kappa of 0.52-0.88 [53,54].
Validity

High concurrent validation with the Visual Analog Scale ( $r=.84$ to .94 , $P<.001)[2,49])$.

Good discriminatory capability between background and peak intensity pain in the oncology population with only $14 \%$ of the 240 patients giving inconsistent evaluations [48]

Good convergent validation with the Facial Affective Scale $(r=.71, P<.01)$. Discriminant validation: correlated with the Functional Disability Inventory $(r=.28, P<.05)$.

Good sensitivity to change over a 2week period (mean change 1.89, $\left.t_{68}=5.30, P<.001\right)$ in children and adolescents after surgery [50].

Factor analysis revealed two distinct factors (ie, pain intensity and interference) accounting for $66 \%$ and $75 \%$ of total variance, respectively [51].

Scores on both scales declined significantly from baseline to follow-up, thus testifying to the responsiveness of the BPI for detecting changes [51]

Discriminant validation: significant increases in CPOT scores during painful compared to nonpainful procedures [52-55].

Criterion validation: moderate correlation with patient self-report of pain intensity $(r=.40-.69, P<.05)[52,53]$.

Convergent validation: moderate correlation with pain unpleasantness $(r=.31, P<.01)$ [52].

Sensitivity of $86 \%$ and a specificity of $78 \%$ for the presence of pain during turning procedures were shown for a CPOT cut-off score $>2$ [56].
Anxiety

NRS (0-10)
0: no anxiety,

10: worst possible anxiety
Individual validity and reliability tests were not conducted to date with the NRS for anxiety, but it has been included in the Edmonton Symptom Assessment System, whose validity and reliability have received support over the past two decades [57].

${ }^{\mathrm{a}}$ NRS: Numeric Rating Scale

${ }^{b}$ BPI: Brief Pain inventory

${ }^{\mathrm{c}}$ CPOT: Critical-Care Pain Observation Tool

\section{Pain Unpleasantness}

The NRS will be used to assess the pain unpleasantness of patients. The unpleasantness dimension of symptom experience refers to the degree to which the person is bothered by the unpleasant symptom [58]. The pain unpleasantness NRS is scored on a scale from 0 to 10 with the anchors "not at all unpleasant" for 0 and "most unpleasant feeling possible" for 10 .

\section{Pain Interference}

An adapted version of the Brief Pain Inventory (BPI) will be used. The BPI is a short pain assessment scale developed to measure the intensity of pain during the last 24 hours (sensory dimension) and the interference of the pain in the patient's life (cognitive dimension) [59,60]. The pain severity items are rated individually on an NRS with 0 assigned to "no pain" and 10 to "worst possible pain." Patients are asked to rate their pain severity at the time of interview (pain now) and the worst pain, the least pain, and average pain during the last 24 hours. The 7 
items of pain interference evaluate the impact of pain on general activity, mood, walking/mobilization, work, relationships, sleep, and enjoyment of life. The item work is not considered relevant in the context of cardiac surgery patients who are hospitalized in the ICU or acute care units and will not be administered. Instead, additional items about coughing, deep breathing, appetite, and concentration will be included; these have been observed to have moderate-to-severe pain interference in postoperative cardiac surgery patients $[4,15,61]$.

\section{Muscle Tension}

The assessment of muscle tension will be based on an ordinal scale derived from the behavioral pain scale Critical-Care Pain Observation Tool (CPOT), which was developed and tested for the assessment of pain in critically ill patients after cardiac surgery [53]. Evaluation of muscle tension is done by performing passive flexion and extension of the upper limbs of patients at rest with a score of 0 being assigned for "no resistance to passive movements," 1 for "resistance to passive movements," and 2 for "strong resistance to passive movements or incapacity to complete them."

\section{Anxiety}

For consistency and because intensity of anxiety is of interest in this study, the NRS will also be used to assess patients' anxiety levels.

\section{Vital Signs}

Means of blood pressure (ie, systolic, diastolic, mean arterial pressure) and heart and respiratory rates will be collected from the ICU bedside monitors for 1 minute at each assessment point.

\section{Data Collection}

Sociodemographic (eg, age, gender) and medical-surgical data (eg, type of cardiac surgery, analgesia) will be collected using standardized data collection sheets. Prior to the delivery of each intervention (hand massage or hand-holding) and before the first data collection for the standard care group, patients will complete a self-administered data collection sheet with 5 short questions using the NRS for the self-report of pain intensity, pain unpleasantness, and anxiety, a body map for identifying the site of pain, and an open-ended question for a description of the quality of pain. The interventionist will be masked to patients' self-reports, which will be accessed only at the end of data collection to verify for missing data. The form will be completed before, immediately after the intervention (hand massage or hand-holding), and 30 minutes later, for a total of 3 data collection points per intervention. Those assigned to standard care will complete the form at similar times. This bundle of assessments will be repeated for each of the 3 interventions. Muscle tension and vital signs will also be evaluated at the same assessment points.

Finally, pain interference will be evaluated using a structured interview using the BPI on the second postoperative day to examine any carry-over effects.

\section{Data Analysis}

The data will be entered in the SPSS software version 22.0 (IBM Corp), and a random subset of data will be used to identify missing or erroneous values. This study aims to use intention-to-treat analysis by including every participant who has been randomized, regardless of group assignment, study withdrawal, or protocol deviations.

Descriptive statistics will be calculated for sociodemographic and medical-surgical data and the baseline scores on all the outcome variables. Group differentiation in sociodemographic and medical-surgical characteristics for participant patients will be investigated using chi-square tests of independence for nominal level variables and one-way analyses of variance (ANOVA) for interval and ratio level variables. If group differences exist, the respective variable will be included as a covariate in the subsequent analyses. Frequencies and percentages of the location and descriptors of pain (ie, sensory dimension of pain) will be calculated for each group and assessment point and used to describe the pain characteristics of participants. Data on pain location and quality will be compared over time.

Repeated measures between ANOVA factors will be used to test for treatment (hand massage, hand-holding, rest), time (before, immediately after, and 30 minutes later), and interaction effect for pain intensity, pain unpleasantness, muscle tension, anxiety, and means of vital signs. This will be run for each intervention and each of these outcome variables. One ANOVA test will be performed for pain interference with the independent variable being group assignment (hand massage, hand-holding, standard care). The main comparison of interest is between the hand massage and standard care group and the difference in means pre- and immediately posttreatment.

\section{Ethical Considerations}

Ethical approval has been granted by the Research Ethics Committee of the study setting in February 2016. This protocol has been independently peer reviewed by McGill University and the Quebec Nursing Intervention Research Network, who funded the study (F242710).

Informed and written consent will be obtained from participants. The study's aim and procedure, risks and benefits, right to refuse participation and withdraw at any time without any repercussions on the care provided, confidentiality of data, randomization process, and lack of financial incentives for participation in the study will be explained to patients. They will be equally informed that there is no guarantee that they will benefit from this study. While there are no known risks associated with hand massage and hand-holding, any harmful effects during these interventions will be noted and reported to the Research Ethics Committee.

\section{Validity and Reliability}

This study follows the most recent Consolidated Standards of Reporting Trials guidelines for nonpharmacological treatments and randomized controlled trials [62] and the Standard Protocol Items: Recommendations for Interventional Trials statement for clinical trial protocols [63].

The tools to be used for the assessment of the study outcomes have established validity and reliability in the population of interest in this study (Table 2). Data collection errors in vital 
signs will be minimized by extracting their means from the bedside ICU monitors, which offer a continuous recording of vital signs. Additionally, the interventionist will be trained on the assessment of muscle tension. To enhance rigor, standardized hand massage and hand-holding will be ensured by training the interventionist on the administration of these therapies and by using a camera to monitor the consistency and fidelity with which each of these interventions is delivered [42].

\section{Results}

Recruitment started the end of April 2016, and to date 34 patients have already been recruited. Of these, 24 patients were randomized and had data collection done as several were medically unstable postoperatively or had their surgery cancelled/postponed. Data collection is expected to be complete by January 2017.

\section{Discussion}

\section{Interpretation}

Pain is one of the most common and severe symptoms cardiac surgery patients experience during their ICU stay. The adverse effects of unrelieved acute postoperative pain are numerous and can be taxing to cardiac surgery patients during their recovery but worryingly also on the long term. There is a general agreement in practice guidelines that multimodal approaches to pain management should be implemented in the ICU [20]. Massage, a complementary nonpharmacological intervention, could play an important role in enhancing pharmacological analgesia and maximizing pain relief in the cardiac surgery patients, and providing rigorous empirical evidence for its use in the ICU is strongly awaited to inform practice guidelines.

In the proposed RCT, eligible and consenting patients will be randomly assigned to either the massage (ie, experimental), hand-holding (ie, active control) or standard care group (ie, passive control). While the administration of pleasant and potentially beneficial interventions [42] can help minimize attrition rates in the experimental and active control groups, the use of standard care with rest only could cause higher withdrawals in this group and subsequently increase the risk of attrition bias. In order to counter such bias and as an incentive to make participation in the study more attractive, patients will be informed that hand massage can be offered to those assigned to the active or passive control groups at the end of data collection, if desired.

A strength of this RCT is the administration of massage in the actual ICU environment and the clinical context of the first 24 hours post-cardiac surgery when monitoring is accrued, thus enabling an evaluation of the effectiveness of this intervention on the patient's pain and related signs and symptoms. However, the same ICU environment could interfere with the delivery of some of the planned hand massages and hand-holding in the selected mode (eg, quiet environment) and dose (ie, 3 times for 20 minutes). In order to monitor these interferences and consider them in the interpretation of study findings, a camera will be used to videorecord the interventions. Nonetheless, even if these interventions can no longer be administered (eg, use of invasive equipment on hands postrandomization), attempts to continue collecting patients' self-reports of symptoms will be prioritized over imputation for missing data.

\section{Limitations}

One of the anticipated limitations of this RCT is the lack of blinding of patients in the standard care group and the respective clinical personnel responsible for their care. Although environmental adjustments will be made, these patients will have already been informed of the 3 trial arms and their implications and could easily recognize their group assignment. Equally, it is expected that the ICU personnel will notice the absence of an active intervention and assume patient assignment to standard care. Given the unlikelihood of blinding related to the standard care group, patients could modify their self-report while the personnel could practice differently compared to when they see hand massage/hand-holding administrations, thereby increasing the risk of performance bias.

Only the cardiac surgery ICU patient population will be included in this RCT, which will limit the generalizability of the findings to other ICU patients. Even so, this study will reveal the effectiveness of massage in this group of ICU patients suffering from intense pain and should be a trigger for further research internationally on the use of this intervention in the ICU in addition to the locally established standard care.

\section{Conclusion}

This funded RCT will unravel the potential benefits to reduce ICU postsurgery pain by the use of massage therapy in cardiac surgery ICU patients compared to hand-holding and standard care. The results of this study will serve to inform clinical practice guidelines with regard to the dose, timing, and technique of massage administration for the relief of acute postoperative pain in the ICU in addition to analgesia. If effective, massage could be easily implemented in ICU practice with little resources to maximize pain relief in the acute postoperative period and prevent the serious adverse consequences of unrelieved pain.

\section{Acknowledgments}

This research protocol was supported by funding from McGill University and the Quebec Nursing Intervention Research Network (F242710). The first author is also supported by doctoral awards from the Fonds de Recherche du Québec-Santé, Quebec Nursing Intervention Research Network, Ministère de l'Enseignement Supérieur de la Recherche et de la Science, and McGill Nursing Collaborative Fellowship. The funding agencies were not involved in the preparation of the research protocol or in the writing of this paper. 


\section{Conflicts of Interest}

None declared.

\section{Multimedia Appendix 1}

Modified Consolidated Standards of Reporting Trials flow diagram. CONSORT E-HEALTH (V1.6).

[PDF File (Adobe PDF File), 975KB-Multimedia Appendix 1]

\section{References}

1. Mueller XM, Tinguely F, Tevaearai HT, Revelly JP, Chioléro R, von Segesser LK. Pain location, distribution, and intensity after cardiac surgery. Chest 2000 Aug;118(2):391-396. [Medline: 10936130]

2. Puntillo K, Weiss SJ. Pain: its mediators and associated morbidity in critically ill cardiovascular surgical patients. Nurs Res 1994;43(1):31-36. [Medline: $\underline{8295837]}$

3. Gélinas C. Management of pain in cardiac surgery ICU patients: have we improved over time? Intensive Crit Care Nurs 2007 Oct;23(5):298-303. [doi: 10.1016/j.iccn.2007.03.002] [Medline: 17448662]

4. Watt-Watson J, Stevens B, Katz J, Costello J, Reid GJ, David T. Impact of preoperative education on pain outcomes after coronary artery bypass graft surgery. Pain 2004 May;109(1-2):73-85. [doi: 10.1016/j.pain.2004.01.012] [Medline: 15082128]

5. Yorke J, Wallis M, McLean B. Patients' perceptions of pain management after cardiac surgery in an Australian critical care unit. Heart Lung 2004;33(1):33-41. [doi: 10.1016/j.hrtlng.2003.09.002] [Medline: 14983137]

6. Asilioglu K, Celik SS. The effect of preoperative education on anxiety of open cardiac surgery patients. Patient Educ Couns 2004 Apr;53(1):65-70. [doi: 10.1016/S0738-3991(03)00117-4] [Medline: 15062906]

7. Young E, Eddleston J, Ingleby S, Streets J, McJanet L, Wang M, et al. Returning home after intensive care: a comparison of symptoms of anxiety and depression in ICU and elective cardiac surgery patients and their relatives. Intensive Care Med 2005 Jan;31(1):86-91. [doi: 10.1007/s00134-004-2495-y] [Medline: 15565363]

8. Vingerhoets G. Perioperative anxiety and depression in open-heart surgery. Psychosomatics 1998;39(1):30-37. [doi: 10.1016/S0033-3182(98)71378-7] [Medline: 9538673]

9. Bauer BA, Cutshall SM, Wentworth LJ, Engen D, Messner PK, Wood CM, et al. Effect of massage therapy on pain, anxiety, and tension after cardiac surgery: a randomized study. Complement Ther Clin Pract 2010 May;16(2):70-75. [doi: 10.1016/j.ctcp.2009.06.012] [Medline: 20347836]

10. Braun LA, Stanguts C, Casanelia L, Spitzer O, Paul E, Vardaxis NJ, et al. Massage therapy for cardiac surgery patients-a randomized trial. J Thorac Cardiovasc Surg 2012 Dec;144(6):1453-1459 [FREE Full text] [doi: 10.1016/j.jtcvs.2012.04.027] [Medline: 22964355]

11. Cutshall SM, Wentworth LJ, Engen D, Sundt TM, Kelly RF, Bauer BA. Effect of massage therapy on pain, anxiety, and tension in cardiac surgical patients: a pilot study. Complement Ther Clin Pract 2010 May;16(2):92-95. [doi: 10.1016/j.ctcp.2009.10.006] [Medline: 20347840]

12. Go AS, Mozaffarian D, Roger VL, Benjamin EJ, Berry JD, Blaha MJ, American Heart Association Statistics Committee and Stroke Statistics Subcommittee. Executive summary: heart disease and stroke statistics-2014 update: a report from the American Heart Association. Circulation 2014 Jan 21;129(3):399-410 [FREE Full text] [doi: 10.1161/01.cir.0000442015.53336.12] [Medline: 24446411]

13. Denault A, Lamarche Y, Rochon A, Cogan J, Liszkowski M, Lebon J, et al. Innovative approaches in the perioperative care of the cardiac surgical patient in the operating room and intensive care unit. Can J Cardiol 2014 Dec;30(12 Suppl):S459-S477. [doi: 10.1016/j.cjca.2014.09.029] [Medline: 25432139]

14. Desai PM. Pain management and pulmonary dysfunction. Crit Care Clin 1999 Jan;15(1):151-166. [Medline: 9929792$]$

15. Choinière M, Watt-Watson J, Victor JC, Baskett Roger JF, Bussières JS, Carrier M, et al. Prevalence of and risk factors for persistent postoperative nonanginal pain after cardiac surgery: a 2-year prospective multicentre study. CMAJ 2014 Apr 15;186(7):E213-E223 [FREE Full text] [doi: 10.1503/cmaj.131012] [Medline: 24566643]

16. Kehlet H, Jensen TS, Woolf CJ. Persistent postsurgical pain: risk factors and prevention. Lancet 2006 May 13;367(9522):1618-1625. [doi: 10.1016/S0140-6736(06)68700-X] [Medline: 16698416]

17. Katz J, Seltzer Z. Transition from acute to chronic postsurgical pain: risk factors and protective factors. Expert Rev Neurother 2009 May;9(5):723-744. [doi: 10.1586/ern.09.20] [Medline: 19402781]

18. Gjeilo KH, Stenseth R, Klepstad P. Risk factors and early pharmacological interventions to prevent chronic postsurgical pain following cardiac surgery. Am J Cardiovasc Drugs 2014 Oct;14(5):335-342. [doi: 10.1007/s40256-014-0083-2] [Medline: 24934698]

19. Azzam PN, Alam A. Pain in the ICU: a psychiatric perspective. J Intensive Care Med 2013;28(3):140-150. [doi: 10.1177/0885066611432417] [Medline: 22232202]

20. Barr J, Fraser GL, Puntillo K, Ely EW, Gélinas C, Dasta JF, American College of Critical Care Medicine. Clinical practice guidelines for the management of pain, agitation, and delirium in adult patients in the intensive care unit. Crit Care Med 2013 Jan;41(1):263-306. [doi: 10.1097/CCM.0b013e3182783b72] [Medline: 23269131] 
21. Chanques G, Jaber S, Barbotte E, Violet S, Sebbane M, Perrigault P, et al. Impact of systematic evaluation of pain and agitation in an intensive care unit. Crit Care Med 2006 Jun;34(6):1691-1699. [doi: 10.1097/01.CCM.0000218416.62457.56] [Medline: $\underline{16625136]}$

22. Dunn C, Sleep J, Collett D. Sensing an improvement: an experimental study to evaluate the use of aromatherapy, massage and periods of rest in an intensive care unit. J Adv Nurs 1995 Jan;21(1):34-40. [Medline: 7897075]

23. Richards KC, Gibson R, Overton-McCoy AL. Effects of massage in acute and critical care. AACN Clin Issues 2000 Feb;11(1):77-96. [Medline: 11040555]

24. Ramesh C, Pai V, Patil N, Nayak B, George A, George L, et al. Effectiveness of massage therapy on post-operative outcomes among patients undergoing cardiac surgery: A systematic review. International Journal of Nursing Sciences 2015 Sep;2(3):304-312 [FREE Full text] [doi: 10.1016/j.ijnss.2015.07.006]

25. Asadizaker M, Fathizadeh A, Haidari A, Goharpai S, Fayzi S. The effect of foot and hand massage on postoperative cardiac surgery pain. Int J Nurs Midwifery 2011;3(10):165-169.

26. Najafi SS, Rast F, Momennasab M, Ghazinoor M, Dehghanrad F, Mousavizadeh SA. The effect of massage therapy by patients' companions on severity of pain in the patients undergoing post coronary artery bypass graft surgery: a single-blind randomized clinical trial. Int J Community Based Nurs Midwifery 2014 Jul;2(3):128-135 [FREE Full text] [Medline: 25349854]

27. Nerbass FB, Feltrim MI, Souza SA, Ykeda DS, Lorenzi-Filho G. Effects of massage therapy on sleep quality after coronary artery bypass graft surgery. Clinics (Sao Paulo) 2010;65(11):1105-1110 [FREE Full text] [Medline: 21243280]

28. Babaee S, Shafiei Z, Sadeghi MM, Nik AY, Valiani M. Effectiveness of massage therapy on the mood of patients after open-heart surgery. Iran J Nurs Midwifery Res 2012 Feb;17(2 Suppl 1):S120-S124 [FREE Full text] [Medline: 23833593]

29. Albert NM, Gillinov AM, Lytle BW, Feng J, Cwynar R, Blackstone EH. A randomized trial of massage therapy after heart surgery. Heart Lung 2009;38(6):480-490. [doi: 10.1016/j.hrtlng.2009.03.001] [Medline: 19944872]

30. Boitor M, Martorella G, Arbour C, Michaud C, Gélinas C. Evaluation of the preliminary effectiveness of hand massage therapy on postoperative pain of adults in the intensive care unit after cardiac surgery: a pilot randomized controlled trial. Pain Manag Nurs 2015 Jun;16(3):354-366. [doi: 10.1016/j.pmn.2014.08.014] [Medline: 26025795]

31. Melzack R. From the gate to the neuromatrix. Pain 1999 Aug;Suppl 6:S121-S126. [Medline: 10491980]

32. Andersson S, Lundeberg T. Acupuncture-from empiricism to science: functional background to acupuncture effects in pain and disease. Med Hypotheses 1995 Sep;45(3):271-281. [Medline: 8569551]

33. Degenhardt BF, Darmani NA, Johnson JC, Towns LC, Rhodes DCJ, Trinh C, et al. Role of osteopathic manipulative treatment in altering pain biomarkers: a pilot study. J Am Osteopath Assoc 2007 Sep;107(9):387-400. [Medline: 17908831]

34. Guirimand F, Le Bars D. Physiology of nociception. Ann Fr Anesth Reanim 1996;15(7):1048-1079. [doi: 10.1016/S0750-7658(96)89477-9] [Medline: 9180983]

35. Bremner JD, Krystal JH, Southwick SM, Charney DS. Noradrenergic mechanisms in stress and anxiety: II. Clinical studies. Synapse 1996 May;23(1):39-51. [doi: 10.1002/(SICI)1098-2396(199605)23:1<39::AID-SYN5>3.0.CO;2-I] [Medline: $\underline{8723134]}$

36. Goddard AW, Ball SG, Martinez J, Robinson MJ, Yang CR, Russell JM, et al. Current perspectives of the roles of the central norepinephrine system in anxiety and depression. Depress Anxiety 2010 Apr;27(4):339-350. [doi: 10.1002/da.20642] [Medline: 19960531]

37. Moyer CA, Rounds J, Hannum JW. A meta-analysis of massage therapy research. Psychol Bull 2004 Jan;130(1):3-18. [doi: 10.1037/0033-2909.130.1.3] [Medline: 14717648]

38. Faul F, Erdfelder E, Lang A, Buchner A. G*Power 3: a flexible statistical power analysis program for the social, behavioral, and biomedical sciences. Behav Res Methods 2007 May;39(2):175-191. [Medline: 17695343]

39. Hattan J, King L, Griffiths P. The impact of foot massage and guided relaxation following cardiac surgery: a randomized controlled trial. J Adv Nurs 2002 Jan;37(2):199-207. [Medline: 11851788 ]

40. Smith CA, Collins CT, Cyna AM, Crowther CA. Complementary and alternative therapies for pain management in labour. Cochrane Database Syst Rev 2006;CD003521(4). [doi: 10.1002/14651858.CD003521.pub2] [Medline: 17054175]

41. Cepeda MS, Africano JM, Polo R, Alcala R, Carr DB. What decline in pain intensity is meaningful to patients with acute pain? Pain 2003 Sep;105(1-2):151-157. [Medline: 14499431]

42. Martorella G, Boitor M, Michaud C, Gélinas C. Feasibility and acceptability of hand massage therapy for pain management of postoperative cardiac surgery patients in the intensive care unit. Heart Lung 2014;43(5):437-444. [doi: 10.1016/j.hrtlng.2014.06.047] [Medline: 25064487]

43. Boutron I, Moher D, Altman DG, Schulz KF, Ravaud P. Methods and processes of the CONSORT Group: example of an extension for trials assessing nonpharmacologic treatments. Ann Intern Med 2008 Feb 19;148(4):W60-W66. [Medline: $\underline{18283201]}$

44. Ezzo J. What can be learned from Cochrane systematic reviews of massage that can guide future research? J Altern Complement Med 2007 Mar;13(2):291-295. [doi: 10.1089/act.2007.13604] [Medline: 17388773]

45. Kutner JS, Smith MC, Corbin L, Hemphill L, Benton K, Mellis BK, et al. Massage therapy versus simple touch to improve pain and mood in patients with advanced cancer: a randomized trial. Ann Intern Med 2008 Sep 16;149(6):369-379 [FREE Full text] [Medline: $\underline{18794556]}$ 
46. Gélinas C, Arbour C, Michaud C, Robar L, Côté J. Patients and ICU nurses' perspectives of non-pharmacological interventions for pain management. Nurs Crit Care 2013 Nov;18(6):307-318. [doi: 10.1111/j.1478-5153.2012.00531.x] [Medline: 24165072]

47. Kolcaba K, Schirm V, Steiner R. Effects of hand massage on comfort of nursing home residents. Geriatr Nurs 2006;27(2):85-91. [doi: 10.1016/j.gerinurse.2006.02.006] [Medline: 16638478]

48. Brunelli C, Zecca E, Martini C, Campa T, Fagnoni E, Bagnasco M, et al. Comparison of numerical and verbal rating scales to measure pain exacerbations in patients with chronic cancer pain. Health Qual Life Outcomes 2010;8:42 [FREE Full text] [doi: 10.1186/1477-7525-8-42] [Medline: 20412579]

49. Ahlers SJG, van Gulik L, van der Veen AM, van Dongen HPA, Bruins P, Belitser SV, et al. Comparison of different pain scoring systems in critically ill patients in a general ICU. Crit Care 2008;12(1):R15 [FREE Full text] [doi: 10.1186/cc6789] [Medline: 18279522$]$

50. Pagé MG, Katz J, Stinson J, Isaac L, Martin-Pichora AL, Campbell F. Validation of the numerical rating scale for pain intensity and unpleasantness in pediatric acute postoperative pain: sensitivity to change over time. J Pain 2012 Apr;13(4):359-369. [doi: 10.1016/j.jpain.2011.12.010] [Medline: 22424915]

51. Gjeilo KH, Stenseth R, Wahba A, Lydersen S, Klepstad P. Validation of the brief pain inventory in patients six months after cardiac surgery. J Pain Symptom Manage 2007 Dec;34(6):648-656. [doi: 10.1016/j.jpainsymman.2007.01.010] [Medline: 17629665]

52. Boitor M, Fiola JL, Gélinas C. Validation of the critical-care pain observation tool and vital signs in relation to the sensory and affective Components of Pain During Mediastinal Tube Removal in Postoperative Cardiac Surgery Intensive Care Unit Adults. J Cardiovasc Nurs 2016;31(5):425-432. [doi: 10.1097/JCN.0000000000000250] [Medline: 25829139]

53. Gélinas C, Fillion L, Puntillo KA, Viens C, Fortier M. Validation of the critical-care pain observation tool in adult patients. Am J Crit Care 2006 Jul;15(4):420-427 [FREE Full text] [Medline: 16823021]

54. Linde SM, Badger JM, Machan JT, Beaudry J, Brucker A, Martin K, et al. Reevaluation of the critical-care pain observation tool in intubated adults after cardiac surgery. Am J Crit Care 2013 Nov;22(6):491-497 [FREE Full text] [doi: 10.4037/ajcc2013700] [Medline: 24186820]

55. Keane KM. Validity and reliability of the critical care pain observation tool: a replication study. Pain Manag Nurs 2013 Dec;14(4):e216-e225. [doi: 10.1016/j.pmn.2012.01.002] [Medline: 24315275]

56. Gélinas C, Harel F, Fillion L, Puntillo KA, Johnston CC. Sensitivity and specificity of the critical-care pain observation tool for the detection of pain in intubated adults after cardiac surgery. J Pain Symptom Manage 2009 Jan;37(1):58-67. [doi: 10.1016/j.jpainsymman.2007.12.022] [Medline: 18599262]

57. Nekolaichuk C, Watanabe S, Beaumont C. The Edmonton Symptom Assessment System: a 15-year retrospective review of validation studies (1991-2006). Palliat Med 2008 Mar;22(2):111-122. [doi: 10.1177/0269216307087659] [Medline: $\underline{18372376]}$

58. Lenz ER, Pugh LC, Milligan RA, Gift A, Suppe F. The middle-range theory of unpleasant symptoms: an update. ANS Adv Nurs Sci 1997 Mar;19(3):14-27. [Medline: 9055027]

59. Cleeland CS, Ryan KM. Pain assessment: global use of the Brief Pain Inventory. Ann Acad Med Singapore 1994 Mar;23(2):129-138. [Medline: 8080219]

60. Larue F, Colleau SM, Brasseur L, Cleeland CS. Multicentre study of cancer pain and its treatment in France. BMJ 1995 Apr 22;310(6986):1034-1037 [FREE Full text] [Medline: 7728056]

61. Martorella G, Côté J, Racine M, Choinière M. Web-based nursing intervention for self-management of pain after cardiac surgery: pilot randomized controlled trial. J Med Internet Res 2012;14(6):e177 [FREE Full text] [doi: 10.2196/jmir.2070] [Medline: 23241361]

62. Boutron I, Moher D, Altman DG, Schulz KF, Ravaud P. Extending the CONSORT statement to randomized trials of nonpharmacologic treatment: explanation and elaboration. Ann Intern Med 2008 Feb 19;148(4):295-309. [Medline: 18283207]

63. Chan A, Tetzlaff JM, Gøtzsche PC, Altman DG, Mann H, Berlin JA, et al. SPIRIT 2013 explanation and elaboration: guidance for protocols of clinical trials. BMJ 2013;346:e7586 [FREE Full text] [Medline: 23303884]

\section{Abbreviations}

ANOVA: analysis of variance

BPI: Brief Pain Inventory

CPOT: Critical-Care Pain Observation Tool

ICU: intensive care unit

NRS: Numeric Rating Scale

RCT: randomized controlled trial 
Edited by G Eysenbach; submitted 28.06.16; peer-reviewed by AR Jung, B Bauer; comments to author 24.08.16; revised version received 19.09.16; accepted 19.09.16; published 07.11.16

Please cite as:

Boitor M, Martorella G, Laizner AM, Maheu C, Gélinas $C$

The Effectiveness of Hand Massage on Pain in Critically Ill Patients After Cardiac Surgery: A Randomized Controlled Trial Protocol JMIR Res Protoc 2016;5(4):e203

URL: http://www.researchprotocols.org/2016/4/e203/

doi: $10.2196 /$ resprot. 6277

PMID: 27821384

CMadalina Boitor, Géraldine Martorella, Andréa Maria Laizner, Christine Maheu, Céline Gélinas. Originally published in JMIR Research Protocols (http://www.researchprotocols.org), 07.11.2016. This is an open-access article distributed under the terms of the Creative Commons Attribution License (http://creativecommons.org/licenses/by/2.0/), which permits unrestricted use, distribution, and reproduction in any medium, provided the original work, first published in JMIR Research Protocols, is properly cited. The complete bibliographic information, a link to the original publication on http://www.researchprotocols.org, as well as this copyright and license information must be included. 